\title{
Shock-related radio emission during coronal mass ejection lift-off? (Research Note)
}

\author{
S. Pohjolainen
}

Tuorla Observatory/Department of Physics, University of Turku, 21500 Piikkiö, Finland

e-mail: silpoh@utu.fi

Received 27 November 2007 / Accepted 20 February 2008

\begin{abstract}
Aims. We identify the source of fast-drifting decimetric-metric radio emission that is sometimes observed prior to the so-called flare continuum emission. Fast-drift structures and continuum bursts are also observed in association with coronal mass ejections (CMEs), not only flares.

Methods. We analyse radio spectral features and images acquired at radio, $\mathrm{H} \alpha$, EUV, and soft X-ray wavelengths, during an event close to the solar limb on 2 June 2003.

Results. The fast-drifting decimetric-metric radio burst corresponds to a moving, wide emission front in the radio images, which is normally interpreted as a signature of a propagating shock wave. A decimetric-metric type II burst where only the second harmonic lane is visible could explain the observations. After long-lasting activity in the active region, the hot and dense loops could be absorbing or suppressing emission at the fundamental plasma frequency. The observed burst speed suggests a super-Alfvénic velocity for the burst driver. The expanding and opening loops, associated with the flare and the early phase of CME lift-off, could be driving the shock. Alternatively, an instantaneous but fast loop expansion could initiate a freely propagating shock wave. The later, complexlooking decametre-hectometre wave type III bursts indicate the existence of a propagating shock, although no interplanetary type II burst was observed during the event. The data does not support CME bow shock or a shock at the flanks of the CME as the origin of the fast-drift decimetric-metric radio source. Therefore super-Alfvénic loop expansion is the best candidate for the initiation of the shock wave, and this result challenges the current view of metric/coronal shocks originating either in the flanks of CMEs or from flare blast waves.
\end{abstract}

Key words. Sun: flares - Sun: coronal mass ejections (CMEs) - Sun: radio radiation - plasmas

\section{Introduction}

Frequency-drifting features at decimetric-metric wavelengths are usually observed in association with flares and coronal mass ejections (CMEs). Some groups of fast-drift bursts within a restricted bandwidth have been identified as type II precursors (Klassen et al. 1999), and interpreted as signatures of reconnection processes above expanding soft X-ray loops that later lead to type II burst emission (Klassen et al. 2003). Type II bursts are generally believed to be formed by propagating shock fronts that accelerate electrons; these electrons excite Langmuir waves, which convert to radio waves observable as plasma emission close to the fundamental and second-harmonic frequencies (Melrose 1980; Cairns et al. 2003). As the burst exciter moves outwards in the solar atmosphere, the plasma density decreases, which causes a frequency-drift to lower frequencies. The highest frequencies of the fundamental components of type II bursts rarely exceed a few hundred $\mathrm{MHz}$ (Lin et al. 2006)

Shocks can be either freely propagating blast waves or driven shocks (piston-driven or bow-shocks ahead of a projectile); for a recent review, see Warmuth (2007). There is an ongoing debate about whether the exciters of metric type II bursts are flarerelated or CME-related (e.g., Cane \& Erickson, 2005). Metric type II bursts are sometimes accompanied by stationary and long-lasting continuum emission, which forms out of the trailing edge of a type II burst (Robinson \& Smerd 1975). These have been classified as type II-related flare continuum bursts (FCII), and the emission is most probably plasma radiation at the fundamental frequency. Flare continuum (FC) emission was introduced by Wild (1970), to separate the strong, early-in-theflare appearing continuum from the stationary type IV continuum that usually appears well after the flare or after the lift-off phase of a CME. To add confusion to this classification scheme, flare continuum can sometimes be followed by a moving type IV burst (i.e., where the source is moving spatially, not just frequency-drifting); a schematic representation is provided by Robinson (1978).

Vourlidas et al. (2007) presented an event that shows a longduration continuum burst at $70-20 \mathrm{MHz}$. This continuum feature fitted well into the FCII classification, since it was associated with a type II burst. Farnik et al. (2003) had already analysed the earlier evolution of the same event, and observed an "unusual drifting continuum" in the $400-40 \mathrm{MHz}$ frequency range, preceding the FCII continuum burst (see Fig. 14 of their paper). They associate the unusual drifting metric continuum with expanding EUV and soft X-ray loops.

We analyse a rare event with a continuum similar to that of a FCII classification, apart from the fact that no interplanetary type II burst is detected. The FCII-like structure was preceded by an unusual fast-drifting decimetric-metric radio feature that started at a very high frequency, close to $1 \mathrm{GHz}$, and later blended with the FCII-like continuum. Since this event occurred close to the solar limb, and imaging at radio, EUV, $\mathrm{H} \alpha$, and soft $\mathrm{X}$-ray wavelengths exist, we attempt to determine the exciter of the fast-drift feature, and to ascertain whether this feature and 

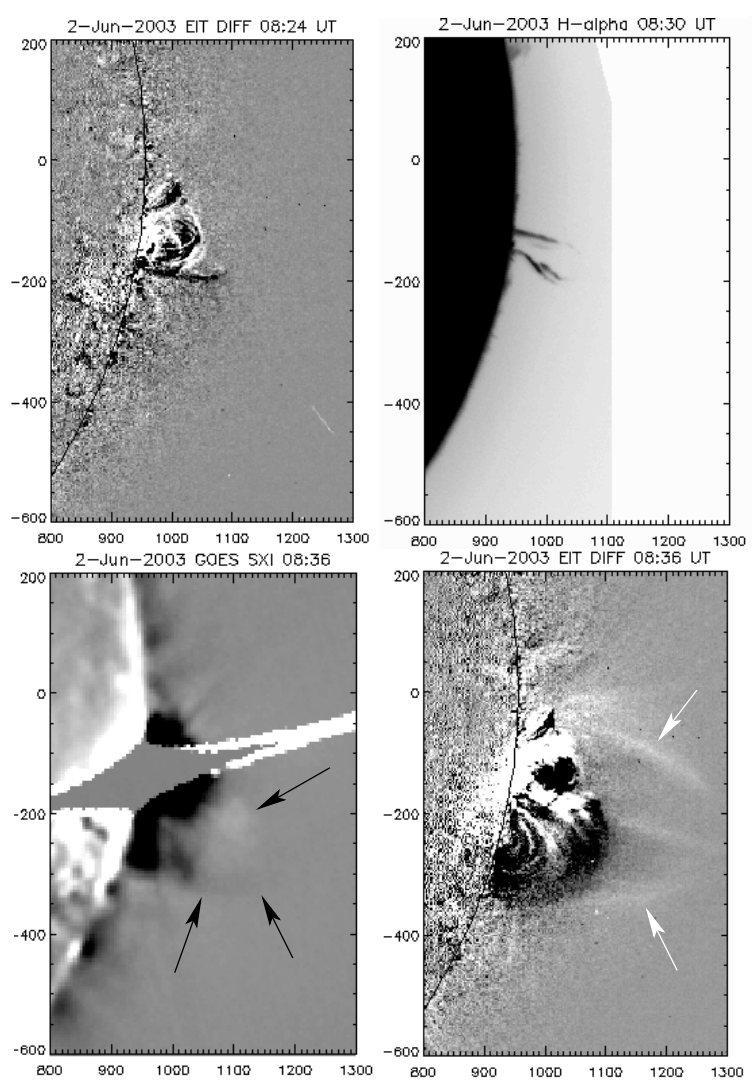
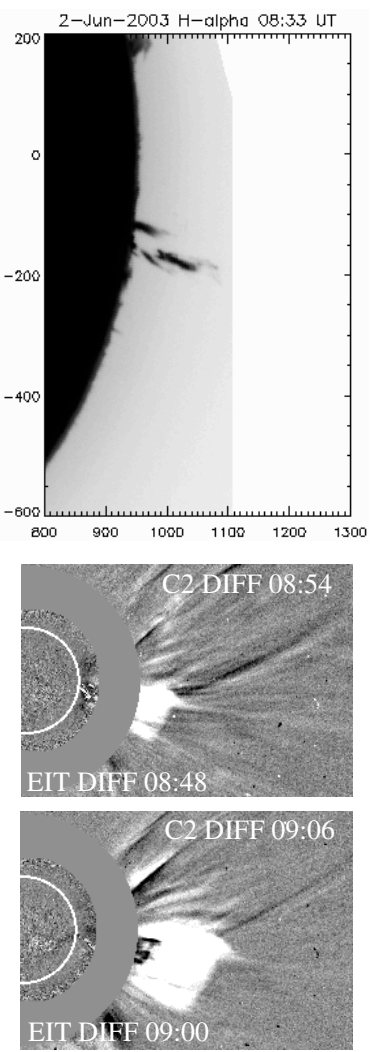

Fig. 1. Evolution of the 2 June 2003 eruption: SOHO EIT difference image at 08:24-08:12 UT, Kanzelhöhe $\mathrm{H} \alpha$ images at 08:30 and 08:33 UT, GOES SXI difference image at 08:36-08:32 UT, SOHO EIT difference image at 08:36-08:12 UT, and SOHO LASCO C2 difference images at 08:54 and 09:06 UT (LASCO CME Catalog). At 08:54 UT, the CME front is already at heliocentric distance $3.1 R_{\odot}$. At 08:36 UT, a bright loop-like structure is observed in soft X-rays (indicated with black arrows) and at the same time bright EUV rays are visible over the active region (white arrows). The hard X-ray experiment RHESSI observed an emission source just above the solar limb at 08:14 UT, but unfortunately the satellite entered night-time one minute later, and no comparison could be made with the hard X-ray data. An $\mathrm{H} \alpha$ flare was recorded at 08:41-08:44 UT (NGDC NOAA flare lists, data not shown here). the FCII-like continuum are related. We also consider the connection with the simultaneously appearing CME.

\section{Observations}

A GOES M3.9 class flare in NOAA AR 10365 (S07 W89) on 2 June 2003 started with a flux rise at 08:12 UT, followed by new rises at 08:21 and 08:27 UT, before the flux maximum at 08:37 UT. Since this was a limb flare, it is possible that the flare was partly occulted and hence the start time and flux evolution are uncertain. A filament eruption started in between the available Kanzelhöhe $\mathrm{H} \alpha$ images at 08:23 and 08:30 UT. From later images, it is possible to estimate the (projected) plane-of-the-sky speed of the filament, which is at least $330 \mathrm{~km} \mathrm{~s}^{-1}$.

SOHO EIT (Delaboudinière et al. 1995) images, starting at 08:24 UT, show the erupting filament, together with loop displacements. In the EIT images at 08:36 and 08:48 UT large-scale changes in the active region are evident. At 08:36 UT, bright EUV structures are observed at height $\approx 1.35 R_{\odot}$, which is higher than the simultaneously observed soft X-ray loop-like structure, observed by GOES SXI near $1.27 R_{\odot}$, see Fig. 1 .

A partial halo-type CME was first observed at 08:54 UT by SOHO LASCO (Brueckner et al. 1995), close to a heliocentric height of $3.1 R_{\odot}$ (Fig. 1). The CME velocity, measured using a linear fit to all height-time data points, was $980 \mathrm{~km} \mathrm{~s}^{-1}$ (LASCO CME Catalog). No strong evidence of acceleration or deceleration in the CME speed was observed. Backward-extrapolation of the CME heights places the CME front close to the solar limb at about 08:30 UT.

The radio dynamic spectrum from Phoenix-2 (Messmer et al. 1999) shows the onset of a fast-drift burst close to $1000 \mathrm{MHz}$ at 08:29 UT (Fig. 2). The frequency drift rate was initially about $2 \mathrm{MHz} \mathrm{s}^{-1}$, but then decreased to a constant value of about $0.7 \mathrm{MHz} \mathrm{s}^{-1}$, between 08:32 UT and 08:37 UT. The narrow emission band and the fast drift suggest that this is plasma radiation. The local plasma frequency (fundamental emission), $f_{\mathrm{p}}$, can be used to estimate the local electron density, $n_{\mathrm{e}}$, as $f_{\mathrm{p}}=9000 \sqrt{n_{\mathrm{e}}}$. Electron density can in turn be converted to height with the use of atmospheric density models. The use of density models, and the uncertainties involved, are explained in detail in, e.g., Pohjolainen et al. (2007).

At 08:31:10 UT, the low-frequency edge of the fast-drift emission lane was located at $500 \mathrm{MHz}$, which corresponds to an electron density of $3.1 \times 10^{9} \mathrm{~cm}^{-3}$ (assuming fundamental emission), and by 08:35:00 UT the emission had drifted to $300 \mathrm{MHz}$ $\left(n_{\mathrm{e}} \approx 1.1 \times 10^{9} \mathrm{~cm}^{-3}\right)$. We assume that the density gradient followed standard atmospheric models, because the frequency drift was almost constant during this time. Since the highest densities are found only in active region loops and streamers, we have to multiply the standard atmospheric models by suitable coefficients. To determine these coefficients, we use observed radio source heights as a constraint. The fast-drift emission sources were imaged at the Nançay Radioheliograph (Kerdraon \& Delouis 1997) frequencies of 432 and $327 \mathrm{MHz}$. The radio images show a wide arc-like front over the western limb, above $1.2 R_{\odot}$ after 08:32 UT (Fig. 3). To reproduce the observed height of $1.2 R_{\odot}$ at $327 \mathrm{MHz}$, we need to use 19-times Saito (Saito 1970) or 8-times Newkirk (Newkirk 1961) model densities. A 19-times Saito model density gives a height of $1.097 R_{\odot}$ for $500 \mathrm{MHz}$, and a height of $1.231 R_{\odot}$ for $300 \mathrm{MHz}$. If we calculate the speed directly from these heights and times, it is about $405 \mathrm{~km} \mathrm{~s}^{-1}$.

The direction of the moving radio arc was towards the Northwest, and it appears as if the front was moving away from the observer. The observed speed must therefore be corrected for projection effects. The observed, projected velocity of the front was approximately $300 \mathrm{~km} \mathrm{~s}^{-1}$, suggesting a "true" deprojected velocity of $400-500 \mathrm{~km} \mathrm{~s}^{-1}$. 


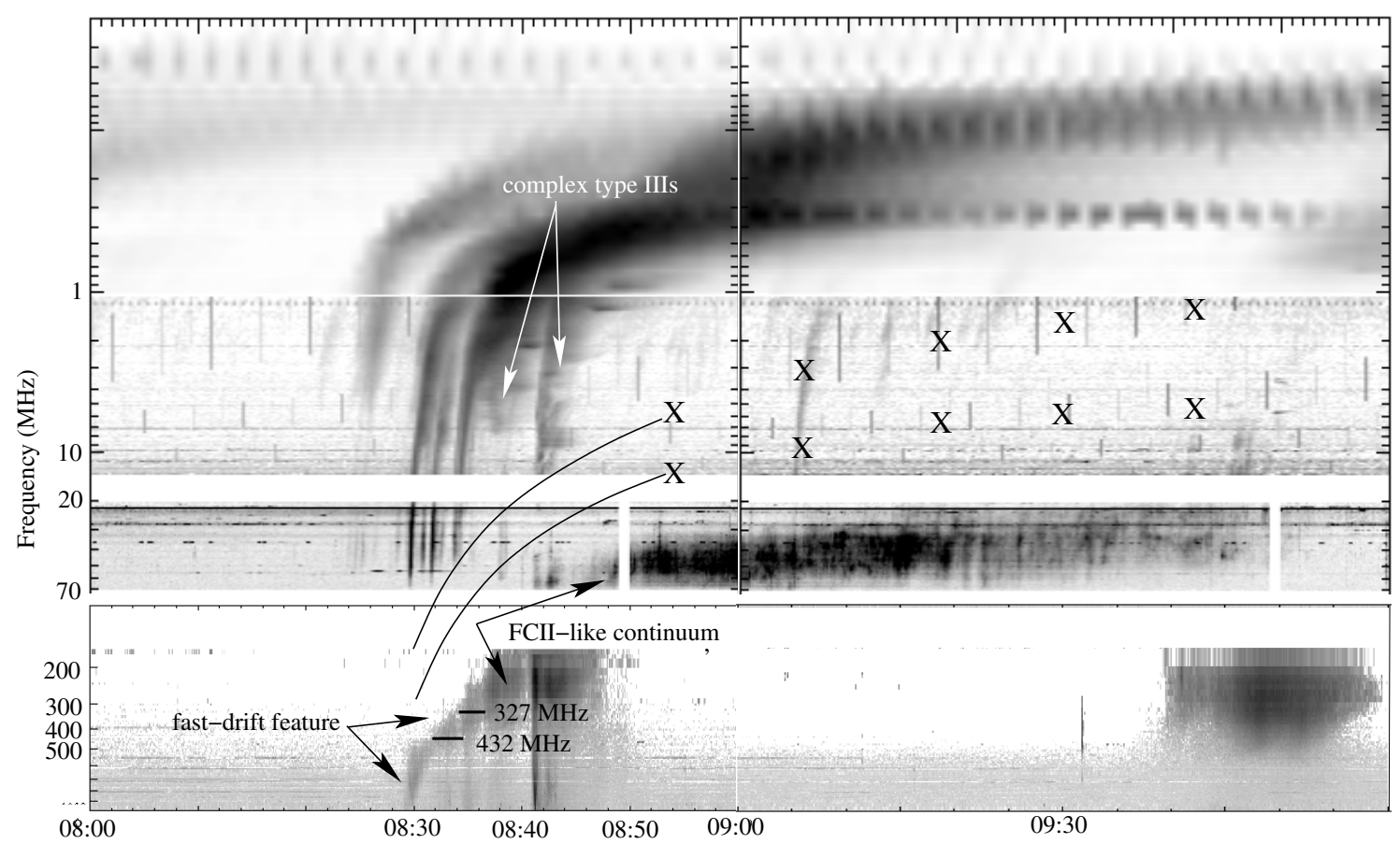

Fig. 2. A fast-drift radio feature was observed on 2 June 2003 from $1000 \mathrm{MHz}$ to around $300 \mathrm{MHz}$, where the emission lane blended with a wide-band FCII-like continuum emission. The continuum feature was best observed at $70-20 \mathrm{MHz}$, and it decreased in intensity close to $10 \mathrm{MHz}$ at about 09:50 UT. Complex-looking type III bursts were observed after 08:36 UT. The "X"s mark the plasma frequencies calculated from the observed heights of the plane-of-the-sky CME front locations, using Saito and 2-times Newkirk atmospheric density models. Solid black lines represent the frequency-time profiles for a disturbance travelling at a speed of $980 \mathrm{~km} \mathrm{~s}^{-1}$. This spectral composite includes observations from Phoenix-2, Nançay Decameter Array, and Wind WAVES.
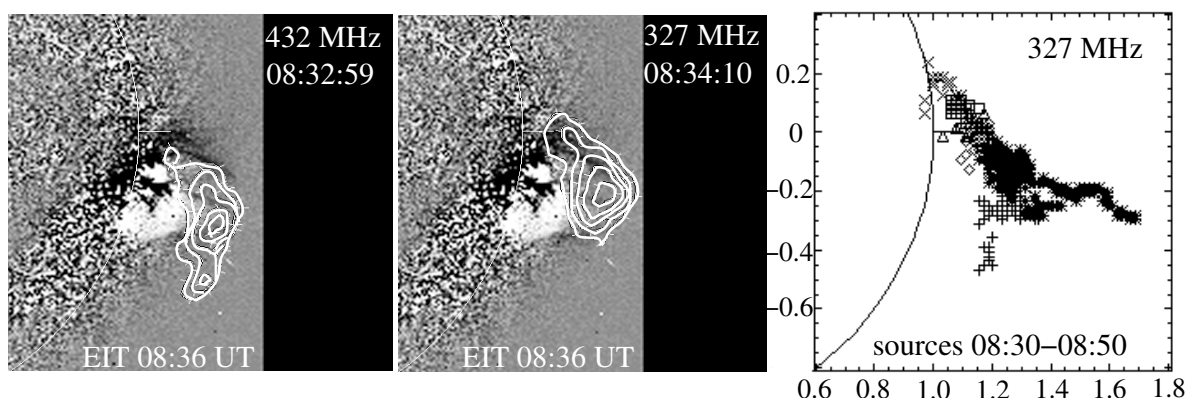

Fig. 3. Contours of the radio emission sources at 08:32:59 UT (432 MHz, left) and 08:34:10 UT (327 MHz, middle), observed during the fast-drift spectral feature (Nancay Radioheliograph imaging). The radio source centroids during 08:30-08:50 UT at $327 \mathrm{MHz}$ (right) show both the fast-drift feature and the FCII-like continuum sources. The EUV image on the background is the EIT difference image with reversed color scale at 08:36 UT (the bright EUV rays show as black). The EIT field of view does not extend beyond $1.4 R_{\odot}$. The three plots all show the same region, and the axes are in solar radiae.
At about 08:36 UT, the emission lane of the fast-drift feature disappeared into a wider continuum emission, starting from close to $300 \mathrm{MHz}$. A slowly-moving emission source is observed in the Nancay Radioheliograph images at $327-150 \mathrm{MHz}$, during 08:42-09:00 UT (Fig. 3). These frequencies are close to the high-frequency edge of the continuum, which was observed best at 70-20 MHz (Nançay Decameter Array observations; Lecacheux 2000). The continuum emission faded away from the spectrum at about 09:50 UT, close to $10 \mathrm{MHz}$ (Wind WAVES observations; Bougeret et al. 1995).

Figure 2 shows the estimated emission frequencies at the CME front, for the case in which the CME creates a bow shock at its leading edge. We note, however, that no interplanetary type II burst was visible at decametre-hectometre wavelengths. The frequencies were calculated using the standard atmospheric density models of Saito and 2-times Newkirk; the use of even higher density models would move the "X"s and the frequency-drift profiles to lower positions in the plot, towards higher frequencies.
Wind WAVES observations show complex-looking radio type III bursts starting around 08:36 UT (Fig. 2). The tilted and disturbed type III burst lanes have earlier been associated with shocks, either by electron beams traversing turbulent shock regions (Reiner et al. 2000; Lehtinen et al. 2008) or by electrons accelerated by the shock (Cane et al. 1981). Either way, and in spite of the fact that no interplanetary type II burst was observed, a propagating shock must have existed.

\section{Results and discussion}

In the analysed event, as in the event described by Farnik et al. (2003), the start of radio emission was preceded by long-term (tens of minutes) activity in X-rays. The outcome of earlier heating would have been that hot and dense loops existed high in the corona. The observed plasma emission would reflect the high densities in these loops, and the high densities explain the high values of starting frequency. The initially fast drift rates could be attributed to the rapidly-changing densities. Radio images of the fast-drift spectral feature show an outward-moving arc-like 
structure. This structure is observed above the limb at heights $>1.2 R_{\odot}$, which is much higher than predicted by any standard atmospheric density model, at these frequencies.

The frequency drifts of metric type II bursts are usually in the range of $0.1-1.0 \mathrm{MHz} \mathrm{s}^{-1}$ (Nelson \& Melrose 1985). The observed frequency drift rate of the fast-drift feature was $\approx 0.7 \mathrm{MHz} \mathrm{s}^{-1}$ between 500 and $300 \mathrm{MHz}$, after the very fast start. The duration of this spectral feature was 6-7 min, which is well within the observed usual durations of metric type II bursts. It is also known that due to plasma processes, emission at the fundamental plasma frequency can be weaker than at the second harmonic at metric wavelengths. For decametre-hectometre waves, the converse occurs. Thus, it is possible that the fast-drift metric radio feature was the second harmonic lane of a type II burst, and due to some (unknown) processes the fundamental emission band was either suppressed or absorbed. Of course, the non-visibility of weaker fundamental lanes can be due to instrumental effects. By adopting the type II interpretation, the fastdrift feature fulfils the definition of a later-appearing FCII continuum, because then it is type II burst-related. The tilted and complex-looking type III bursts also indicate the presence of a shock wave.

If we assume that the observed emission was emission at the second harmonic, the corresponding fundamental plasma frequencies are $250 \mathrm{MHz}$ and $150 \mathrm{MHz}$. Using generally-accepted 10 -times Saito model densities, these frequencies provide atmospheric heights of 1.19 and $1.37 R_{\odot}$, respectively. These values are close to the observed source heights and imply a burst speed of $545 \mathrm{~km} \mathrm{~s}^{-1}$. The calculations in Sect. 2 provide a burst speed of $405 \mathrm{~km} \mathrm{~s}^{-1}$; to obtain this value, we need to assume 19-times Saito atmospheric densities if emission occurs at the fundamental plasma frequency. Both of these values are still in agreement with the $400-500 \mathrm{~km} \mathrm{~s}^{-1}$ de-projected speed of the imaged radio arc.

What is the origin of the shock responsible for the type II emission? In the vicinity and high above active regions, where the densities are high but the magnetic field strengths are low, the local magnetosonic (Alfvén) speed can be as low as $200 \mathrm{~km} \mathrm{~s}^{-1}$; see for example Warmuth \& Mann (2005). A super-Alfvénic shock is then possible. Ejected material or high-speed loop expansion could drive the shock. Freely-propagating blast waves could accelerate the electrons required to produce a type II burst, although these waves would die out sooner than the driven, super-Alfvénic waves. The bright EUV rays, above the soft $\mathrm{X}$-ray loop, alludes to an overlying arcade that opens during CME formation; the rays would then represent compressed material at the flanks. However, a shock formed at the flank of a CME would be unlikely to display a wide, moving radio arc. No $\mathrm{H} \alpha$ Moreton wave (a blast wave signature) was visible during this event, although at the limb they are difficult to detect. No loop ejection was observed either. Therefore super-Alfvénic loop expansion is the best candidate for the initiation of the shock wave, and this result challenges the current view that metric/coronal shocks originate either at the flanks of CMEs or from flare blast waves.

The frequency-time profiles for the later-observed whitelight CME front do not agree with the evolution of the fast-drift decimetric-metric feature. The frequency-time track of the CME is, however, similar to the frequency drift of the FCII-like continuum. Kai (1969) reported an event in which the radio images at $80 \mathrm{MHz}$ show arc-like radio emission, followed closely by an outward-moving single source. The single moving source was then classified as a moving type IV burst. Since our FCII-like continuum source shows movement outwards, the assumption that it is a "stationary flare continuum source" can also be questioned.

Since the analysis of high-frequency, fast-drift features requires both wide frequency range spectral and radio imaging observations combined with multi-wavelength high time-cadence imaging, the results presented here are preliminary and require comparison to other similar - but rare - events.

Acknowledgements. SOHO is a project of international cooperation between ESA and NASA. GOES SXI is operated by the National Oceanic and Atmospheric Administration and their data is available at the NGDC website. The LASCO CME Catalog is generated and maintained at the CDAW Data Center by NASA and The Catholic University of America in cooperation with the Naval Research Laboratory, and it is available at http://cdaw.gsfc. nasa.gov/CME_list/. Radio data was accessed with the help of the Radio Monitoring Survey at http://secchirh.obspm.fr/select.php, generated and maintained at the Observatoire de Paris by the LESIA UMR CNRS 8109 in cooperation with the Artemis team, Universities of Athens and Ioanina and the Naval Research Laboratory. The solar radio group at LESIA is thanked for the Nancay Radioheliograph data. The Phoenix-2 dynamic radio spectral data are available at the ETHZ Radio Astronomy and Plasma Physics group website. Thanks are due to Vasyl Yurchyshyn for providing the $\mathrm{H} \alpha$ data (Global HighResolution $\mathrm{H} \alpha$ Network, of which Kanzelhöhe Observatory is a member). The comments and suggestions from the referee improved the paper significantly. Rami Vainio is thanked for discussions and careful reading of the manuscript.

\section{References}

Bougeret, J.-L., Kaiser, M. L., Kellogg, P. J., et al. 1995, Space Sci. Rev., 71, 231 Brueckner, G. E., Howard, R. A., Koomen, M. J., et al. 1995, Sol. Phys., 162, 357

Cairns, I. H., Knock, S. A., Robinson, P. A., \& Kuncic, Z. 2003, Space Sci. Rev., 107, 27

Cane, H. V., \& Erickson, W. C. 2005, ApJ, 623, 1180

Cane, H. V., Stone, R. G., Fainberg, J., et al. 1981, Geophys. Res. Lett., 12, 1285 Delaboudinière J.-P., Artzner, G. E., Brunaud, J., et al. 1995, Sol. Phys., 162, 291 Farnik, F., Hudson, H. S., Karlicky, M., \& Kosugi, T. 2003, A\&A, 399, 1159 Kai, K. 1969, Sol. Phys., 10, 460

Kerdraon A., \& Delouis J.-M. 1997, in Coronal Physics from Radio and Space Observations, ed. G. Trottet, Lect. Notes Phys. (Springer), 483, 192

Klassen, A., Aurass, H., Klein, K.-L., Hofmann, A., \& Mann, G. 1999, A\&A, 343, 287

Klassen, A., Pohjolainen, S., \& Klein, K.-L. 2003, Sol. Phys., 218, 197

Lecacheux, A. 2000, in Radio Astronomy at Long Wavelengths, ed. R. G. Stone, K. W. Weiler, M. L. Goldstein, \& J.-L. Bougerot, AGU Monograph Series, 119,321

Lehtinen, N. J., Pohjolainen, S., Huttunen-Heikinmaa, K., et al. 2008, Sol. Phys., 247,151

Lin, J., Mancuso, S., \& Vourlidas, A. 2006, ApJ, 649, 1110

Melrose, D. B. 1980, Space Sci. Rev., 26, 3

Messmer, P., Benz. A. O., \& Monstein, C. 1999, Sol. Phys. 187, 335

Nelson, G. J., \& Melrose, D. B. 1985, in Solar Radiophysics: studies of Emission from the Sun at Metre Wavelengths, ed. D. J. McLean, \& N. R. Labrum (Cambridge: Cambridge Univ. Press), 333

Newkirk, G. Jr. 1961, ApJ, 133, 983

Pohjolainen, S., van Driel-Gesztelyi, L., Culhane, J. L., Manoharan, P. K., \& Elliott, H. A. 2007, Sol. Phys., 244, 167

Reiner, M. J., Karlický, M., Jiřička, K., et al. 2000, ApJ, 530, 1049

Robinson, R. D. 1978, Aust. J. Phys., 31, 533

Robinson, R. D., \& Smerd, S. F. 1975, PASAu, 2, 374

Saito, K. 1970, Ann. Tokyo Astr. Obs., 12, 53

Vourlidas, A., Pick, M., Hoang, S., \& Demoulin, P. 2007, ApJ, 656, L105

Warmuth, A., \& Mann, G. 2005, A\&A, 435, 1123

Warmuth, A. 2007, in The High Energy Solar Corona: Waves, Eruptions, Particles, ed. K.-L. Klein, \& A. L. MacKinnon, Lect. Notes Phys., 725, 107 (Springer)

Wild, J. P. 1970, PASAu, 1, 365 